\title{
Analyze Angle of the Camera Payload LSU-02 in Mission of Aerial Photo
}

\author{
Sunar ${ }^{1, *}$, Ari Sugeng Budiyanta ${ }^{2}$,Prasepvianto Estu Broto ${ }^{3}$, Agus Bayu Utama $^{4}$ \\ 1,2,3,4 LAPAN, Aeronautics Technology Center, Indonesia
}

\begin{abstract}
In carrying out its mission, LAPAN Surveillance UAV version 2 (LSU-02) was equipped with a camera payload sensor mounted on a gimbal system. When taking upright aerial photos according to photogrammetric requirements, the axis of the aerial camera must be aligned with the direction of gravity with a slope tolerance less than 3 degrees $\left(<3^{\circ}\right)$, so that the shooting results meet the map to the desired standard. The camera payload was equipped with a 3-axis gimbal system which pitch and roll directions installed by the gyro sensor to measure the camera's tilt angle. Gimbal camera payload with dimensions (160x170x155) $\mathrm{mm}$ are designed to be installed on a casing (190x190x180) $\mathrm{mm}$ according to the available space in the LSU-02 payload space, which was the length $\mathrm{x}$ width $\mathrm{x}$ height (190x190x180) mm. Testing was done by flying LSU-02 on Rumpin and Pamengpeuk runway. From the test results, it was obtained the deviation of roll and pitch angle less than 3 degree, which the gimbal angle oscillation when the payload camera shooting was only \pm 1 degree with the respon time until it reaches a stable condition of approximately 35 to 55 seconds, and the offset value for roll and pitch approximately -1 degree, which it has met the standard for making aerial photo maps according to photogrammetric requirements.
\end{abstract}

Keywords: gimbal, payload, upright aerial photo, pitch angle, roll angle, LSU-02

\section{INTRODUCTION}

The aerial mapping technique with photogrammetric approach using UAVs with low cost spatial data collection has been carried out (Muneza J. Maurice, et $a l$ ) [1]. Several aerial photography techniques have been used by using UAV technology and some applications: UAV stabilization techniques during flight by maintenance of a constant speed, maintenance of a straight line of flight and the reduction of tilt (Volodymyr Hlotov et al., 2015)[2]; Oblique photogrammetric system by integrating a cheap doublecamera imaging system designed to acquire images with ground resolutions better than $3 \mathrm{~cm}$ has been conducted by San Jiang et al., 2017 [3],[9]; the methodology for the determination of mapping costs is developed and the example of efficiency evaluation is presented which is based on the photogrammetric data gained by UAV technology using an experimental object has been developed by Lina Kukliene et al., 2017 [4]; Mingxing Gao and his colleagues have conducted high-resolution UAV data use research with digital elevation models (DEM) to identify the shift of the earth's plates to assess seismic hazards (Mingxing Gao et al., 2017) [5]; use of aerial photographs using uav to produce orthophoto maps (D. Wierzbicki et al., 2015) [6]; use of multi-rotor uavs with high resolution camera to produce aerial photographs with a spatial resolution of 2 inches / pixels (Wenang Anurogo et al., 2017) [7]; UAVs used for a wide variety of applications in plant ecology, including mapping vegetation over small-to medium-sized regions (Mitchell B. Cruzan et al., 2016) [10].

Aerial photography is a photograph taken from the air using an air camera mounted on an airplane from a certain height. Aerial photography can be divided into: 1). Upright aerial photography (vertical aerial photography), which is an aerial photograph produced from shooting aerial photographs upright, meaning that the execution of shooting with the optical axis of the camera is really upright or almost upright (in this paper slope tolerance $<3$ degrees), 2). Slanted aerial photos, which are aerial photographs generated from the result of skewed aerial photography, meaning that it is carried out with the optical axis of an aerial camera that forms an angle with a vertical line. There are two types of oblique aerial photography, namely: 1. Angled shooting, which done with the camera forming a small angle towards the vertical direction, 2. Very oblique shooting, which shooting with a camera that forms a very large angle towards the vertical direction. In other words, tilting when the photo is visible. Aerial photography shall not be undertaken when the angle is less than thirty (30) degrees above the horizon [8]. In general, the photos used for maps are upright photos (Wolf, 1974).

One of the variants of the LAPAN Surveillance UAV (LSU) is LSU-02, which is a type of variant that is the mainstay of the survey mission, aerial photography and mapping. In carrying out its mission, UAV Surveillance LAPAN version 2 (LSU-02) was equipped with a camera sensor mounted on a gimbal system. When taking upright aerial photos according to photogrammetric requirements, the axis of the aerial

*Corresponding author: sunar.st@gmail.com 
camera must be aligned with the direction of gravity with a slope tolerance of less than 3 degrees so that the shooting results meet the map to the desired standard. In this paper discusses the use of gimbal systems for mounting the camera sensor at LSU-02 to get a pitch and roll angle deviation of no more than 3 degrees when performing aerial photo operations, so that it meets the photogrammetric requirements for creating aerial photo maps.

\section{METHOD}

\subsection{UAV used}

LSU-02 used in this research shown in figure 1, with the gimbal specifications and camera payload shown in Table 1.

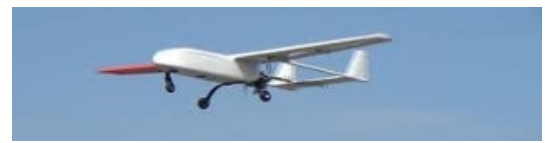

Fig. 1. LSU-02

Table 1.LSU-02 specifications and camera payload installed.

\begin{tabular}{|c|c|}
\hline Element & Specifications \\
\hline LSU-02 & $\begin{array}{ll} & \text { Wing span }=2.400 \mathrm{~mm}(2,4 \mathrm{~m}) \\
\circ & \text { Length of fuselage }=1.700 \mathrm{~mm}(1,7 \\
\mathrm{m}) \\
\circ \text { MTOW }=15 \mathrm{~kg} \\
\circ \text { Weight of payload }=3 \mathrm{~kg} \\
\circ \text { Engine power }=10 \mathrm{Hp} \quad(5 \mathrm{ltr} \\
\text { gasoline fuel capacity }) \\
\circ \text { Max. cruise speed }=100 \mathrm{~km} / \mathrm{h} \\
\circ \text { Endurance }=5 \text { jam } \\
\circ \text { Telecomunication: } 900 \mathrm{MHz}, \quad 1 \\
\text { watt for telemetry, }\end{array}$ \\
\hline $\begin{array}{l}\text { Gimbal } \\
\text { system }\end{array}$ & $\begin{array}{c}\text { DYS } 3 \text { axis Alexmos gimbal } \\
\text { controller }\end{array}$ \\
\hline $\begin{array}{l}\text { Payload } \\
\text { Camera }\end{array}$ & Sony Alpha 6000 \\
\hline
\end{tabular}

\subsection{Gimbal and Payload System}

\subsubsection{Gimbal controller}

Gimbal controller used DYS 3 Axis Brushless

gimbal controller which has the following specifications and features:

Specifications:

- Size: Approx. 52x55mm/2.05×2.17"

- Mounting Hole Diameter: $3 \mathrm{~mm} / 0.12^{\prime \prime}$

- Power Supply Voltage: 8-25 V

- Maximum Motor Current: 1.5 A

- 5V Output Current to Power External Devices: Up to $1 \mathrm{~A}$

Features:

- Upgradeable 32Bit BGC V3.0, with a protection case

- 32bit MCU-ARM Cortex M4. Effectively calculates the complex tasks for 3-axes stabilization
- Allows camera control with the RC or analog joystick

- Use several switchable profiles for different modes of operation

- Supports variety of RC protocols: PWM, SumPPM, spektrum and s-bus

- Battery voltage monitoring, compensating voltage drop in the PID-regulator

- Low battery alarm (output to 5V active buzzer)

- Increased number of inputs for controlling signals + 3 additional reserved input/output AUX1-AUX3

- Can supply up to 1A current for external devices on the $5 \mathrm{~V}$ power line

- Reverse-polarity protection, overheat and overcurrent protection

- USB interface for the PC connection, to configure, control and upgrade firmware

- Control through the Serial-protocol using dedicated API

- Optional second IMU, that will increase the shortterm precision of the stabilization $10 \mathrm{x}-30 \mathrm{x}$ times and will make stable work in any frame position

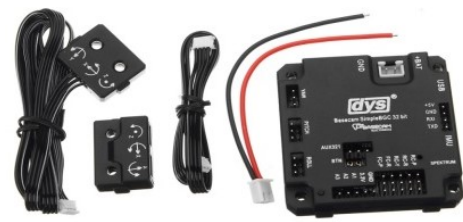

Fig. 2. DYS 3 Axis Brushless Gimbal Controller

This gimbal controller has a feature to control the 3 -axis, but in this study it was only used to control the direction of the 2 axis, the direction of pitch and roll, so that the yaw axis of the gimbal was removed, and the direction of the yaw gimbal follows the direction of the yaw plane.

\subsubsection{Gimbal Mounting and Actuators}

Gimbal mounting and actuators used DYS BLG3SN 3-Axis Brushless Gimbal with 3pcs BGM4108-130 Brushless Motors for Sony NEX.

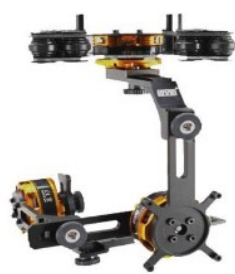

Fig. 3. DYS BLG3SN 3-Axis Brushless Gimbal with 3pcs BGM4108-130 Brushless Motors

\section{Specifications:}

- Material: Aluminum alloy \& carbon fiber on the top.

- Camera: Support Sony NEX / Lumix GH or equivalent weight and size (Alpha 6000)

- Gimbal Weight: 328g(not included motors)

- Gimbal Motor: BGM4108-130 x3

- Wire Weight: $150 \mathrm{~g}$

- $\Omega \mathrm{Ri}: 17.0 \mathrm{ohm}$ 


\section{- Motor Weight: $93 \mathrm{~g}$}

\subsubsection{Integration Gimbal System in LSU-02}

Gimbal controller, gimbal mounting along with a brushless motor, and the camera sensor was placed on the casing as the mounting, then it placed into the casing placement chamber in the fuselage so that it was integrated in the LSU-02 aircraft as shown in figure 4.

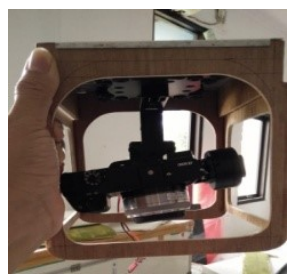

4(a) : Casing, Gimbal and camera

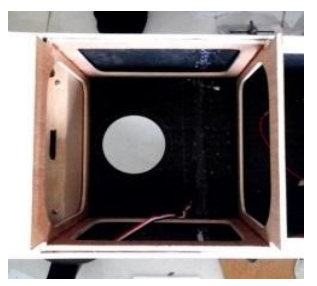

4(b) : Gimbal casing placement space in fuselage

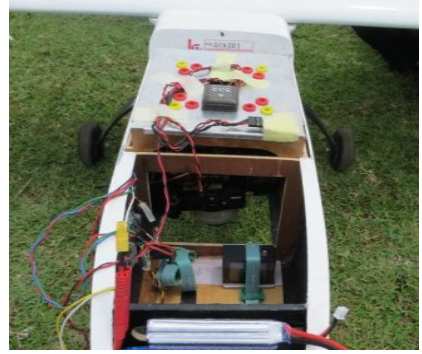

(c): payload integrated in fuselage

Fig. 4. Integration of camera payload system into fuselage LSU-02

\subsection{Data Processing}

To plan the flyway using mission planner software [11]. Data processing was done by according steps;first, storing data pitch and roll of camera angle behavior during flight operation (in logfile in memory), second, downloading data into *.xls files, then plotting in graphical be interpreted until conclusions were obtained.

\section{RESULTS AND DISCUSSION}

The LSU-02 aircraft was flown $500 \mathrm{~m}$ altitude from the ground surface with a programmed waypoint trajectory with an average cruising distance of $1.5 \mathrm{~km}$ per track for testing on the Rumpin runway as shown in figure 5 and tested with an average cruising distance $10 \mathrm{~km}$ trajectory for each track for testing on the Pamengpeuk runway as shown in figure 9. Analysis was done when the cruising flight position, because at this position the best shooting results were produced and in accordance with the conditions of taking aerial photography data carried out by the aircraft while carrying out aerial photo missions.

\subsection{Test Results on Rumpin Runway}

Tests on the Rumpin runway, carried out 2 times the flight. From the results of the logfile data and plotting into the graph, the pitch and roll angle data are obtained. The first test results of gimbal payload can be seen in Figure 6.

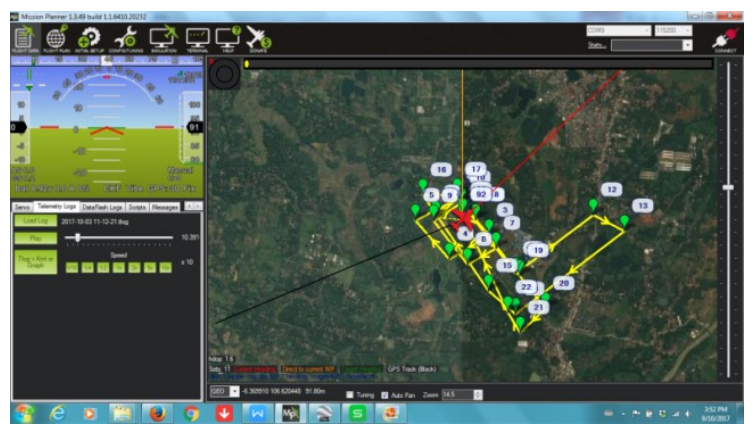

Fig. 5. Flyway test of payload gimbal on Rumpin runway

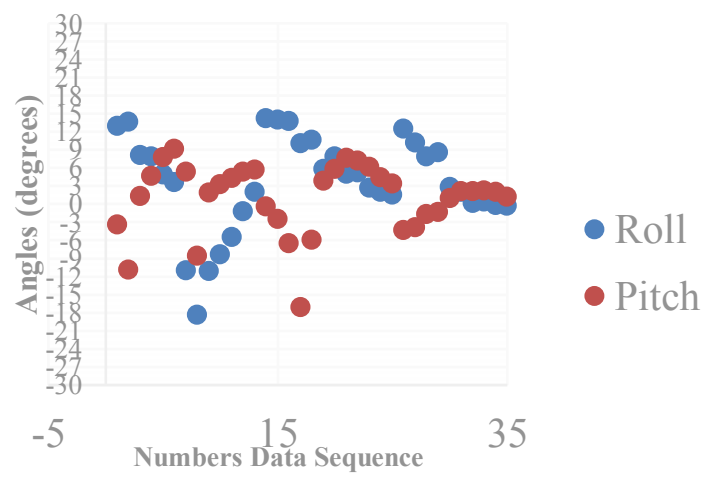

Fig. 6.Angles position of the Roll and Pitch of the payload gimbal when flown

It can be seen from figure 6 that the roll and pitch deviation was still large, which can be up to \pm 12 degrees. Itwas because the engine vibration and installation of the center of gravity (CG) camera affect the stability of the camera angle. Therefore, before being flown back, it was necessary to test the effect of engine vibration on the performance of gimbal. The LSU-02 engine was turned on and then the angle of the camera gimbal deviation was measured at various engine speeds. Engine speed was monitored on the remote controller's Pulse Width Modulation (PWM) scale. The engine speed was increased from PWM values 1000 to 1400 . It seen that the measurement of gimbal angle rises up to a maximum of 1150 PWM and decreases at equal and more than 1300 PWM as can be seen in Figure 7 (a), (b), (c), (d). From the graph, it can be seen that pitch stability (blue line) was better than roll (red line). This was 
presumably because CG roll was less good than CG pitch. For this reason, changes in the mounting arm of the gimbal was made so that the CG roll can be adjusted better. After CG roll was repaired the second flight test was carried out.

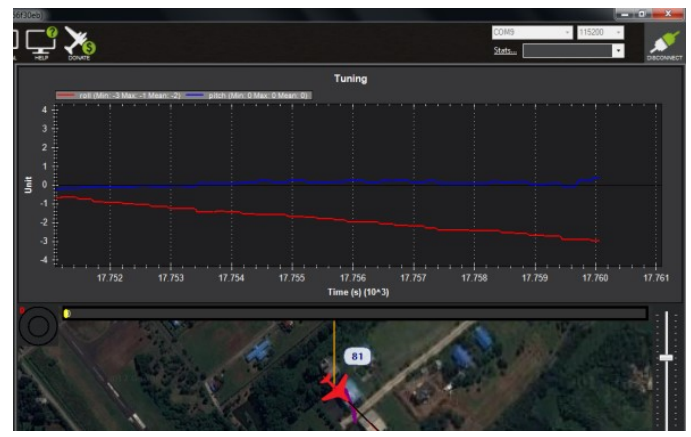

(a) the range of PWM values increased from 1000 to 1150

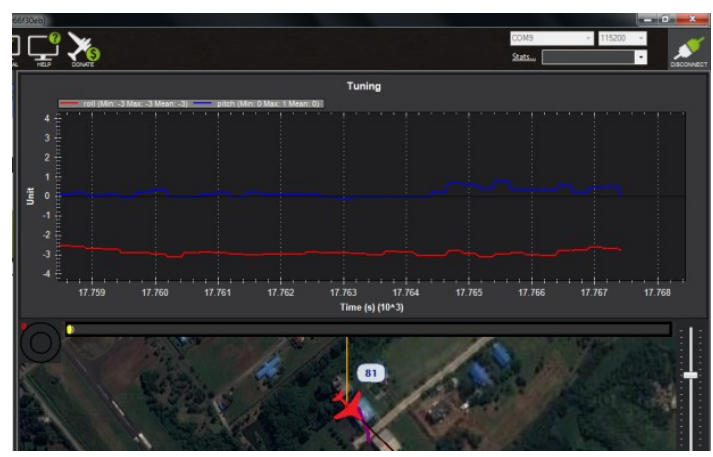

(b) the PWM engine value remains at 1150

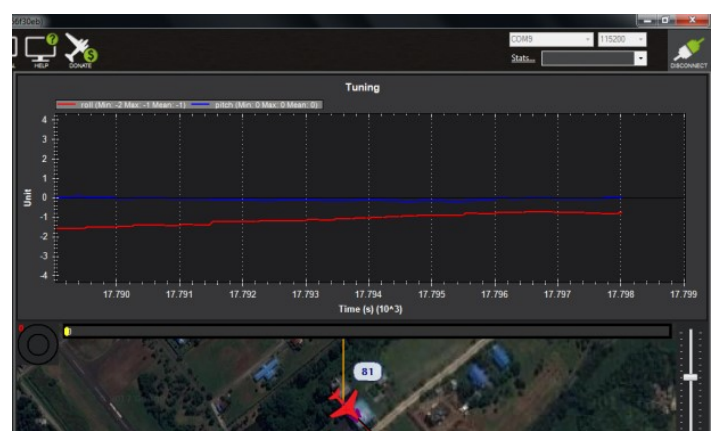

(c) the range of PWM values is increased from 1150 to 1300

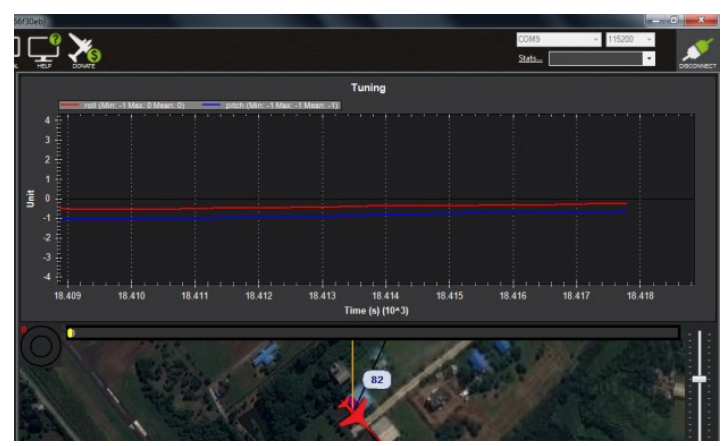

(d) the PWM engine value remains at 1300

Fig.7. Effect of the PWM engine value due to camera gimbal stability

The second test, the plane was flown at an altitude of $500 \mathrm{~m}$ from the ground surface with the same trajectory as the first test. The second test results can be seen in Figure 8. It can be seen from Figure 8 that the roll and pitch deviation is still relatively large, which can be up to \pm 12 degrees when the initial data sequence, but when the data sequence is more than 30 the roll and pitch deviation is less than \pm 3 degrees.

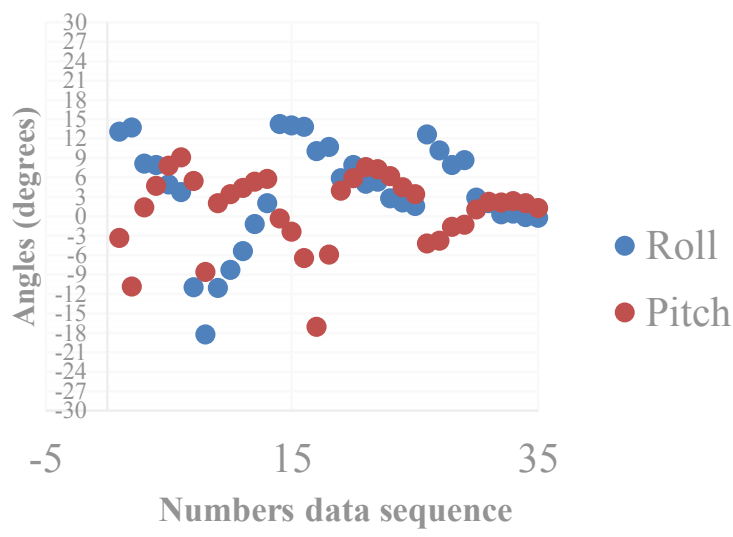

Fig. 8. Angle position of Roll and Pitch of Gimbal

\subsection{Test Results on Pemengpeuk Runway, Garut}

Before flying test, the flight path was programmed as shown in figure 9 . The test was carried out 3 times. The test results were expected to show the performance of the gimbal in adjusting so that the camera was always facing down with an angle less than 3 degrees.

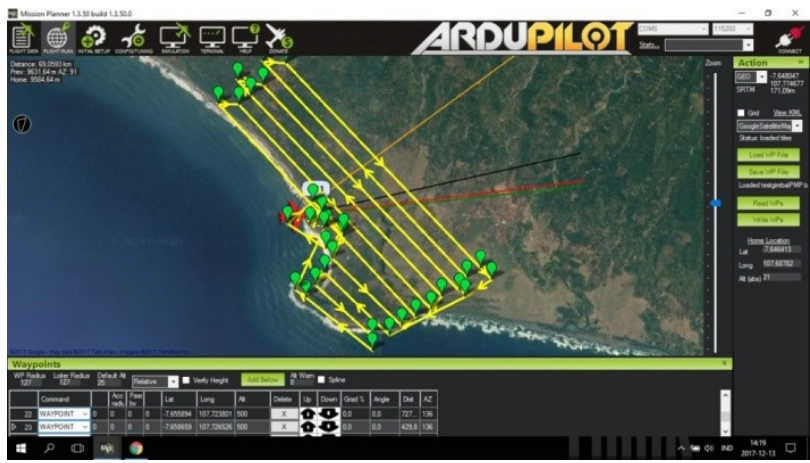

Fig. 9. Flyway testing gimbal-payload LSU-02 in Pamengpeuk

Payload gimbal test on the first day used 2 axis gimbal for roll and pitch directions, so it was expected that aircraft movements in these two directions can be controlled by gimbal to always face down. The test results could be seen in figure 10 . When the Yaw angle 
of the plane showed a constant value, the value of Roll and Pitch of gimbal showed stability in 10 to 15 camera data retrieval. Data is taken every 100 meters, with an average aircraft speed of $100 \mathrm{~km} /$ hour. Camera gimbal reaction to a stable condition of approximately 35 to 55 seconds. While the Roll and Pitch angle oscillations range from \pm 1.5 to 2 degrees and the offset of each Roll and Pitch angle was 6.5 and 3 degrees. To overcome the rsults above, then the test will be carried out on one axis gimbal, namely the gimbal that moves on the roll direction only. The results of camera recording on gimbal concluded that the gimbal vibration still looked quite large.

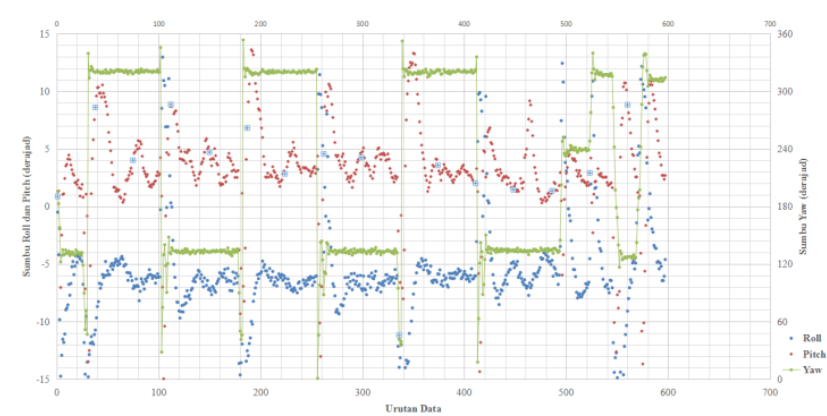

Fig. 10. Results of the first flying gimbal test

The second day test used the same trajectory but used gimbal with one axis in roll direction, while the pitch axis is turned off, so that the payload gimbal pitch axis was the same as the plane's pitch axis. This was done because from the test data the motion of the plane in the direction of the pitch axis showed good results. Figure 11 showed a graph of aircraft movements during cruising. The roll and pitch values each oscillate with a maximum amplitude of 12 and 7 degrees. From the test results, one axis gimbal vibration observed using a relative camera shows a decrease in vibration. Data on camera roll and pitch position when cruising recorded by pixhawk when taking pictures shows oscillations around \pm 1 degree, see figure 12. It showed good enough results because the requirement of photogrammetric mapping was \pm 3 degrees. However, the graph still shows an offset of data of approximately 4 degrees on the roll and 2 degrees on the pitch. While when taking photo data on the first day, when LSU-02 before take off, the plane observed at the level position was 0.49 degrees in the direction of roll, -0.39 degrees in the direction of pitch. So that it can be concluded when the cruise shifts or offset the gimbal direction of several degrees. To overcome this, an attempt was made to check and improve so that the camera's payload mounts on the gimbal precisely at the center of gravity (CG) position of the aircraft.

In addition to monitoring the gimbal payload angle position, monitoring of the angle behavior of the LSU02 aircraft when cruising was shown in Figure 11.

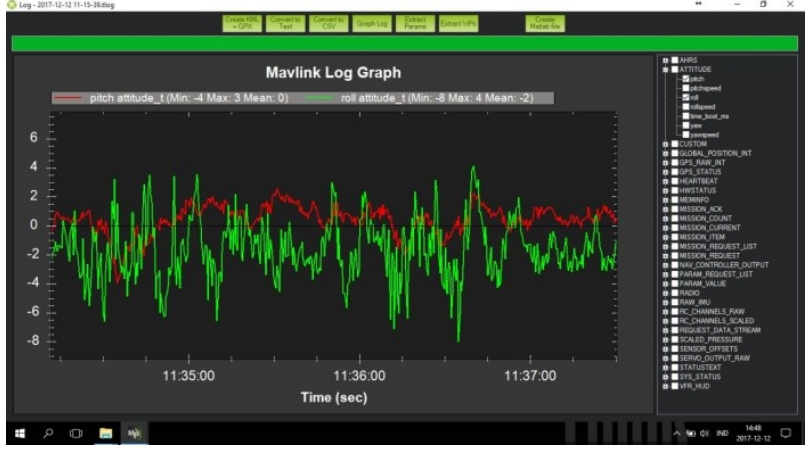

Fig. 11. Monitoring aircraft's roll and pitch angle position during the second flight cruising

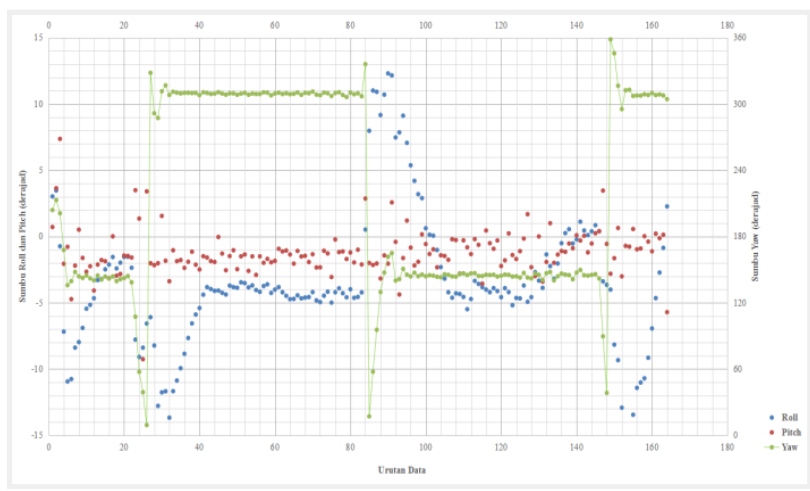

Fig. 12. Results of the second flying payload gimbal test

The results of the third day's test was shown in figure 13. From the graph it can be seen that the position of the camera when shooting has shown a smaller offset value than the results of the second previous test. The offset value for roll and pitch was approximately -1 degree. While the initial value for roll and pitch when each level was 0.12 and 0.00 degrees. For the gimbal oscillation value when shooting was only \pm 1 degree. However, the position adjustment of the gimbal in the direction of the roll still looks slow until the condition reached stable, approximately 35 to 55 seconds (there was a time delay response).

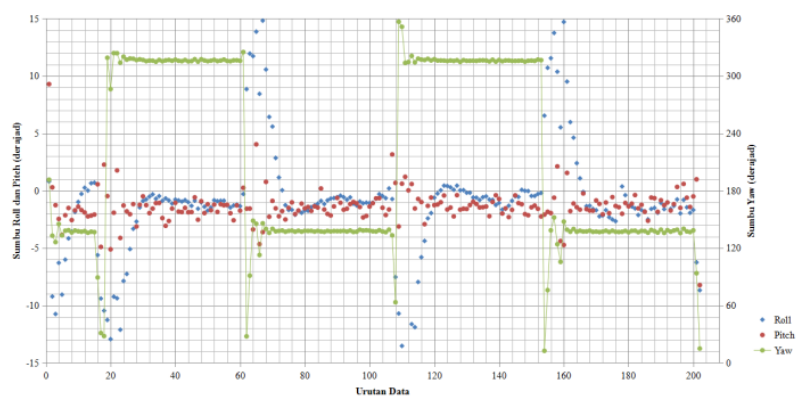

Fig. 13. Results of the third day payload gimbal test

\section{CONCLUSION}

Analysis of camera payload gimbal stability has been carried out at LSU-02 when in Mission of Aerial Photo Operation by analyzing camera angular deviation to gravitational upright lines. Tests were carried out in 2 places, first on the Rumpin runway and second on the 
Pamengpeuk runway. The aircraft was flown at an altitude of $500 \mathrm{~m}$ with a flying route following the programmed waypoint, which is a horizontal route length of $1.5 \mathrm{~km}$ per track in Rumpin, while the length of the track is $10 \mathrm{~km}$ per track in Pamengpeuk. Tests at Rumpin were carried out in 2 flights, the result of the first flight the roll and pitch angle deviation was still large, which could be up to \pm 12 degrees due to engine vibration and the improper installation of center of gravity $(\mathrm{CG})$ cameras, while the second test results after adjusting the PWM engine deviation value roll and pitch anglewere smaller than \pm 3 degree range. To get more valid results, testing was done in Pamengpeuk. Testing in Pamengpeuk is carried out with 3 flights; The results of the first flight of Roll and Pitch angle oscillations ranged from \pm 1.5 to 2 degrees, offset by Roll and Pitch angles of 6.5 degrees and 3 degrees respectively. To overcome the above, then the test will be carried out on one axis gimbal, namely the gimbal that moves on the roll direction only. The second flight was carried out with a one-axis roll gimbal, where the pitch axisof the payload gimbal was same as the plane's pitch axis. The result of the second flight was the camera's roll and pitch angle oscillation when taking a picture of about \pm 1 degree, however the data offset was still about 4 degrees on the roll and 2 degrees on the pitch. To overcome the offset, an attempt was made to check and fix the mounting of the camera's payload gimbal so that it was right in the center of gravity (CG) position of the aircraft, then the third flight test continued. The result of the third flight of gimbal angle oscillation when shooting was only \pm 1 degree, with response time until it reaches a stable condition of approximately 35 to 55 seconds, and the offset value for roll and pitch was approximately -1 degrees. It can be concluded that the stability of the payload gimbal camera of LSU-02 when performing aerial photo missions can meet the photogrammetry requirement.

\section{ACKOWLEDGMENT}

I would like to thank you to Head of the Aeronautical Technology Center and the dissemination team who provided the opportunity in the LSU-02 payload research. Thanks alot of also for the INSINAS Ristekdikti 2018 fiscal year program which has provided financial support in this study.

\section{References}

1. Muneza J. Maurice, Mila N. Koeva, Markus Gerke, Francesco Nex ,Caroline Gevaert, A photogrammetric approach for map updating using UAV in Rwanda, GeoTechRwanda 2015 - Kigali (2015)

2. Volodymyr Hlotov, Zbigniew Siejka, Vadym Kolesnichenko, Oleksandr Prokhorchuk, Anatoliy Tserklevych, Lyubov Babiy, The Analysis of The
Results of Aerial Photography Experiments on The Basis of a Developed UAV Model, Nr IV/3/2015, POLSKA AKADEMIA NAUK, Oddział w Krakowie, s. 1329-1350, Komisja Technicznej Infrastruktury Wsi (2015).

3. San Jiang, Wanshou Jiang, Wei Huang and Liang Yang, UAV-Based Oblique Photogrammetry for Outdoor Data Acquisition and Offsite Visual Inspection of Transmission Line, Remote Sens., 9, 278 (2017)

4. Lina Kukliene, Dainora Jankauskiene, Indrius Kuklys, Birute Ruzgiene, Requirements for Aerial Mapping Using UAV-photogrammetry Technology: Baltic Sea Coast Measurement, International Scientific Conference "RESEARCH FOR ENVIRONMENT AND CIVIL ENGINEERING DEVELOPMENT 17" Proceedings "CIVIL ENGINEERING`17”'(2017).

5. Mingxing Gao, Xiwei $\mathrm{Xu}$, Yann Klinger, Jerome van der Woerd \& Paul Tapponnier, High-resolution mapping based on an Unmanned Aerial Vehicle (UAV) to capture paleoseismic offsets along the Altyn-Tagh fault, China, Scientific Reports, www.nature.com/scientificreports (2017)

6. D. Wierzbicki, M. Kedzierski, A. Fryskowska, Assesment of the Influence of UAV Image Quality on the Orthophoto Production, The International Archives of the Photogrammetry, Remote Sensing and Spatial Information Sciences, Volume XL1/W4, 2015

7. Wenang Anurogo et al., A Simple Aerial Photogrammetric Mapping System Overview and Image Acquisition Using Unmanned Aerial Vehicles (UAVs), Journal of Applied Geospatial Information, Vol 1 No 1, 2017

8. Department of Transportation The State of New Jersey, Minimum Guidelines for Aerial Photogrammetric Mapping, p. 1-1

9. I. Aicardi, F. Chiabrando, N. Grasso, A.M. Lingua, F. Noardo, A. Spano, UAV Photogrammetry with Oblique Images: First Analysis on Data Acquisition and Processing, The International Archives of the Photogrammetry, Remote Sensing and Spatial Information Sciences, Volume XLI-B1, Prague, Czech Republic, 2016

10. Mitchell B. Cruzan, Ben G. Weinstein, Monica R. Grasty, Brendan F. Kohrn, Elizabeth C. Hendrickson, Tina M. Arredondo, and Pamela G. Thompson, Small unmanned aerial vehicles (micro- 
UAVs, drones) in plant ecology, Applications in Plan Sciences, http://www.bioone.org/loi/apps , Botanical Society of America, 2016

11. , Mission Planner Home, http://ardupilot.org/planner/ (accessed Oktober 2018) 